Honam Mathematical J. 34 (2012), No. 2, pp. 199-208

http://dx.doi.org/10.5831/HMJ.2012.34.2.199

\title{
ON SPACES WHICH HAVE COUNTABLE TIGHTNESS AND RELATED SPACES
}

\author{
Woo Chorl Hong
}

\begin{abstract}
In this paper, we study some properties of spaces having countable tightness and spaces having weakly countable tightness. We obtain some necessary and sufficient conditions for a space to have countable tightness. And we introduce a new concept of weakly countable tightness which is a generalization of countable tightness and show some properties of spaces having weakly countable tightness.
\end{abstract}

\section{Introduction}

All spaces under considered here are always assume to be infinite and $T_{1}$. Our terminology is standard and follows [4] and [15].

We recall some definitions which will be used in this paper.

The tightness [2] of $X$ is the smallest infinite cardinal $\tau$ such that for any subset $A$ of $X$ and any point $x \in X$, there exists a subset $B$ of $X$ for which $|B| \leqq \tau$ and $x \in \bar{B}$. Countable tightness is an important concept in topological spaces. A space having countable tightness (also called closure countable [15] or c-space [11]) has been studied by several authors (see [2],[6],[7],[8],[11] and [12]). A space $X$ is sequential [5] if and only if for each subset $A$ of $X$ which is not closed in $X$, there are $x \in \bar{A} \backslash A$ and a sequence $\left(x_{n}\right)$ of points of $A$ such that $\left(x_{n}\right)$ converges to $x$ in $X$. It is well-known that a countable space as well as a space in which every subspace is separable has countable tightness, every first countable space is sequential and every sequential space has countable tightness. But, the converses need not be true in general (see [1],[2],[5],[7],[8] and [12]).

Received February 27, 2012. Accepted March 19, 2012.

2000 Mathematics Subject Classification. 54A20, 54C10, 54D50, 54D55, and $54 \mathrm{D} 99$.

Key words and phrases. countable tightness, sequential, strongly sequentially closed, sequentially closed, weakly countable tightness, $k$-spaces, and $k q$-spaces. 
A subset $A$ of a space $X$ is sequentially closed [5] if and only if no sequence of points of $A$ converges to a point not in $A$. A subset $U$ of a space $X$ is sequentially open [5] if and only if each sequence of points of $X$ converging to a point in $U$ is eventually in $U$. It is well-known that a space $X$ is sequential if and only if every sequentially closed set in $X$ is closed if and only if every sequentially open set in $X$ is open (see [5] and $[8])$.

A sequence $\left(x_{n}\right)$ of points of a space $X$ accumulates to a point $x$ in $X$ [4] if and only if for each open set $U$ in $X$ containing $x$ and each $m \in \mathbb{N}$, there exists $n \in \mathbb{N}$ such that $m \leqq n$ and $x_{n} \in U$; that is, for each open set $U$ in $X$ containing $x,\left(x_{n}\right)$ is frequently in $U$. A subset $A$ of a space $X$ is a strongly sequentially closed [8] set in $X$ if and only if no sequence of points of $A$ accumulates to a point not in $A$. A subset $A$ of a space $X$ is a strongly sequentially open [8] set in $X$ if and only if each sequence of points of $X$ accumulating to a point in $A$ is frequently in $A$.

Recently, in [8], the author showed that every open (closed) set is strongly sequentially open (strongly sequentially closed) and every strongly sequentially open (strongly sequentially closed) set is sequentially open (resp. sequentially closed) and the converses need not be true in general. And he prove that a space $X$ has countable tightness if and only if every strongly sequentially open set in $X$ is open if and only if every strongly sequentially closed set in $X$ is closed.

For each subset $A$ of a space $X$, let

$[A]_{c}=\{x \in X: x \in \bar{C}$ for some countable subset $C$ of $A\}$,

$[A]_{\text {seq }}=\left\{x \in X:\left(x_{n}\right)\right.$ converges to $x$ in $X$ for some sequence $\left(x_{n}\right)$ of points of $A\}$.

The functions $[\cdot]_{c}$ and $[\cdot]_{\text {seq }}$ from the power set $\mathfrak{P}(X)$ of $X$ into $\mathfrak{P}(X)$ itself are called the c-closure operator [8] and the sequential closure operator [1] on $X$, respectively.

It is clear that the $c$-closure operator $[\cdot]_{c}$ satisfies the Kuratowski closure axioms (see [8]). However, the sequential closure operator $[\cdot]_{\text {seq }}$ satisfies the Kuratowski closure axioms except for idempotency in general (see [1],[7] and [8]).

Obviously, for each subset $A$ of a space $X, A \subset[A]_{\text {seq }} \subset[A]_{c} \subset \bar{A}$ and for each countable subset $C$ of $X,[C]_{c}=\bar{C}$.

Note that a subset $A$ of a space $X$ is strongly sequentially closed (sequentially closed) if and only if $A=[A]_{c}$ (resp. $A=[A]_{\text {seq }}$ ) and a space $X$ has countable tightness (is sequential) if and only if for each subset $A$ of $X,[A]_{c}=\bar{A}$ (resp. $A=[A]_{\text {seq }}$ implies $A=\bar{A}$ ) (see [8]). 
In this paper, we study some properties of sequential spaces, spaces having countable tightness and spaces having weakly countable tightness. We obtain some necessary and sufficient conditions for a space to have countable tightness and for a space to be sequential, respectively. And we introduce a new concept of weakly countable tightness which is a generalization of countable tightness. We show the following:

Let $X$ be a countably compact and submaximal space. Then $X$ has countable tightness if and only if $X$ is a $k$-space having weakly countable tightness.

Every closed subspace of a space having weakly countable tightness has weakly countable tightness.

Let $f: X \rightarrow Y$ be a continuous, open and surjective function from a space $X$ having weakly countable tightness onto a space $Y$ satisfying for each open set $U$ in $X, f^{-1}(f(U))=U$. Then $Y$ has weakly countable tightness.

\section{Results}

We begin by characterizing sequentially closed sets and strongly sequentially closed sets.

Lemma 2.1. ([9, Lemma 2.2]) Let $X$ be a Hausdorff space. A subset $A$ of $X$ is sequentially closed if and only if for each sequence $\left(x_{n}\right)$ in $X$ converging to a point $x$ of $X, A \cap\left(\left\{x_{n}: n \in \mathbb{N}\right\} \cup\{x\}\right)$ is closed, where $\left\{x_{n}: n \in \mathbb{N}\right\}$ is the range of $\left(x_{n}\right)$.

Note that a space $X$ is sequential if and only if for a subset $A$ of $X$, $A=[A]_{\text {seq }}$ implies $A=\bar{A}$.

Theorem 2.2. A necessary and sufficient condition for a Hausdorff space $X$ to be sequential is that if for each subset $A$ of $X$ and each sequence $\left(x_{n}\right)$ in $X$ converging to a point $x$ of $X, A \cap\left(\left\{x_{n}: n \in \mathbb{N}\right\} \cup\{x\}\right)$ is closed, then $A$ is closed.

Proof. It follows immediately from Lemma 2.1.

Lemma 2.3. A subset $A$ of a space $X$ is strongly sequentially closed if and only if for each countable subset $C$ of $A, A \cap \bar{C}$ is closed.

Proof. Since $A$ is strongly sequentially closed, $A=[A]_{c}$ and hence for each countable subset $C$ of $A, \bar{C} \subset A$. Thus $A \cap \bar{C}=\bar{C}$ and so it is closed. 
Conversely, let $A$ be a subset of $X$ such that for each countable subset $C$ of $A, A \cap \bar{C}$ is closed. It is enough to show that $A=[A]_{c}$. Then for each countable subset $C$ of $A, C \subset A \cap \bar{C}$ and so $\bar{C} \subset \overline{A \cap \bar{C}}$. By hypothesis, $A \cap \bar{C}$ is closed and hence $\bar{C} \subset A \cap \bar{C}$. Thus $\bar{C} \subset A$ and therefore $A=[A]_{c}$.

Lemma 2.4. ([8, Theorem 2.11]) A necessary and sufficient condition for a space $X$ to have countable tightness is that every strongly sequentially closed subset of $X$ is closed.

Theorem 2.5. A necessary and sufficient condition for a space $X$ to have countable tightness is that if for each subset $A$ of $X$ and each countable subset $C$ of $A, A \cap \bar{C}$ is closed, then $A$ is closed.

Proof. It follows from Lemmas 2.3 and 2.4 .

Consider the following property of a space $X$.

(*) For each countable subset $C$ of $X$ and each subset $A$ of $\bar{C}, \bar{A}=[A]_{c}$.

Note that a space $X$ satisfies property $(*)$ if and only if for each countable subset $C$ of $X$, the subspace $\bar{C}$ of $X$ has countable tightness.

Since countable tightness is hereditary, it is clear that every space having countable tightness satisfies property $(*)$. But, the converse need not be true in general as shown by examples below.

Example 2.6. The space of ordinals $X=\left[0, \omega_{1}\right]$, where $\omega_{1}$ is the first uncountable ordinal, is a Hausdorff compact space. It is clear that for each countable subset $C$ of $X$, the subspace $\bar{C}$ of $X$ has countable tightness. Hence, $X$ satisfies property $(*)$. But, it is well-known that $X$ does not have countable tightness.

Example 2.7. Let $X$ be an uncountable set and $p$ a particular point of $X$. Define a topology for $X$ as follows. For each $x \in X$ with $x \neq p$, $\{x\}$ is open. A subset $U$ of $X$ with $p \in U$ is open if and only if $X \backslash U$ is countable. Then the space $X$ is Hausdorff (see [13, Fortissimo space]). And $X$ satisfies property $(*)$. For, let $C$ be a countable subset of $X$, obviously $C$ is closed and hence for each subset $A$ of $\bar{C}, A$ is countable. Thus $A$ is closed and so $A=[A]_{c}=\bar{A}$. Therefore, $X$ satisfies property $(*)$. But, $X$ does not have countable tightness. For, $p \in \overline{X \backslash\{p\}}$ and there does not exist a countable subset $C$ of $X \backslash\{p\}$ such that $p \in \bar{C}$.

Theorem 2.8. If for each countable subset $C$ of a space $X,|\bar{C} \backslash C| \leqq$ $\aleph_{0}$, then $X$ satisfies property $(*)$. 
Proof. Let $C$ be a countable subset of a space $X$. Then by hypothesis; $|\bar{C} \backslash C| \leqq \aleph_{0}, \bar{C}$ is countable and hence the subspace $C$ of $X$ has countable tightness. Thus, $X$ satisfies property $(*)$.

Note that the space $X$, in Example 2.6, satisfies the condition that for each countable subset $C$ of $X,|\bar{C} \backslash C| \leqq \aleph_{0}$, but $X$ does not have countable tightness. And we know that the converse of Theorem 2.8 is not true. For, the real line $R$ with the usual topology has countable tightness and $|\bar{Q} \backslash Q|>\aleph_{0}$, where $Q$ is the set of all rational numbers.

We recall that a space $X$ is submaximal [3] if and only if every subset of $X$ is open in its closure in $X$ or, equivalently, is the intersection of an open subset and a closed subset of $X$. It is well-known that a space $X$ is submaximal if and only if for every subset $A$ of $X, \bar{A} \backslash A$ is closed if and only if for every subset $A$ of $X, \bar{A} \backslash A$ is discrete if and only if for each subset $A$ of $X, \bar{A} \backslash A$ is closed and discrete if and only if every dense subset of $X$ is open if and only if every subset of $X$ with empty interior is closed and discrete (see [3] and [10]).

Lemma 2.9. For each subset $A$ of a countably compact and submaximal space $X,|\bar{A} \backslash A|<\aleph_{0}$.

Proof. Since $X$ is submaximal, for each subset $A$ of $X, \bar{A} \backslash A$ is closed and discrete (see [10, Theorem 3.2]). Since $X$ is countably compact and $\bar{A} \backslash A$ is closed, $\bar{A} \backslash A$ is countably compact. Note that since every infinite subset of a countably compact space has a limit point, every infinite discrete space is not countably compact. Thus $|\bar{A} \backslash A|<\aleph_{0}$.

Theorem 2.10. Every countably compact and submaximal space $X$ satisfies property $(*)$.

Proof. It follows from Theorem 2.8 and Lemma 2.9.

Lemma 2.11. Let $X$ be a space satisfying property (*). A subset $A$ of a space $X$ is strongly sequentially closed if and only if for each countable subset $C$ of $X, A \cap \bar{C}$ is closed.

Proof. Let $B=A \cap \bar{C}$. Then since $A$ is strongly sequentially closed and $C$ is countable, $B=A \cap \bar{C}=[A]_{c} \cap[C]_{c}$ and so $[B]_{c}=\left[[A]_{c} \cap[C]_{c}\right]_{c}$. We now show that $\left[[A]_{c} \cap[C]_{c}\right]_{c}=[A]_{c} \cap[C]_{c}$. Let $x \in\left[[A]_{c} \cap[C]_{c}\right]_{c}$. Then there exists a countable subset $D$ of $[A]_{c} \cap[C]_{c}$ such that $x \in[D]_{c}$. Since $D \subset[A]_{c}$ and $D \subset[C]_{c}$ and $D$ is countable, $x \in[D]_{c} \subset\left[[A]_{c}\right]_{c}=[A]_{c}$ and $x \in[D]_{c} \subset\left[[C]_{c}\right]_{c}=[C]_{c}$ because the $c$-closure operator $[\cdot]_{c}$ satisfies the Kuratowski closure axioms. Hence, $x \in[A]_{c} \cap[C]_{c}$ and thus it holds. 
Return to the proof, from the fact above we have $[B]_{c}=[A]_{c} \cap[C]_{c}$ and so $[B]_{c}=B$. Since $B \subset \bar{C}$, by property $(*),[B]_{c}=\bar{B}$. Thus $B$ is closed.

Conversely, let $A$ be a subset of $X$ such that for each countable subset $C$ of $X, A \cap \bar{C}$ is closed. It is sufficient to show that $A=[A]_{c}$; equivalently, for each countable subset $B$ of $A,[B]_{c} \subset A$. Clearly, $B$ is also a countable subset of $X$ and hence by hypothesis, $A \cap \bar{B}$ is closed. Since $B \subset A \cap \bar{B}$ and $A \cap \bar{B}$ is closed and $B$ is countable, it follows that $[B]_{c}=\bar{B} \subset A \cap \bar{B}$ and thus $[B]_{c} \subset A$.

Theorem 2.12. Let $X$ be a space satisfying property $(*)$. A necessary and sufficient condition for a space $X$ to have countable tightness is that if for each subset $A$ of $X$ and each countable subset $C$ of $X, A \cap \bar{C}$ is closed, then $A$ is closed.

Proof. It follows from Lemmas 2.4 and 2.11.

Corollary 2.13. Let $X$ be a countably compact and submaximal space. A necessary and sufficient condition for a space $X$ to have countable tightness is that if for each subset $A$ of $X$ and each countable subset $C$ of $X, A \cap \bar{C}$ is closed, then $A$ is closed.

Proof. It follows from Theorems 2.10 and 2.12.

We recall that a subset $A$ of a space $X$ is a $k$-closed set [9] if and only if for each compact subset $K$ of $X, A \cap K$ is closed. A space $X$ is a $k$-space $[9,14](k q$-space [9]) if and only if every $k$-closed set is closed (resp. every sequentially closed set is $k$-closed). In [9], S. Lin and C. Zheng showed that every sequential space is a $k$-space as well as a $k q$ -space and a space $X$ is sequential if and only if $X$ is a $k$-space and a $k q$-space.

We now introduce a new property of a space which is a generalization of countable tightness.

Definition 2.14. A space $X$ has weakly countable tightness if and only if every strongly sequentially closed set is k-closed.

From definitions and Lemmas and Theorems above, we have easily the following implications.

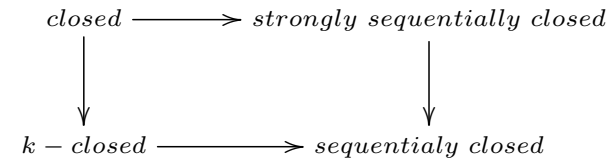




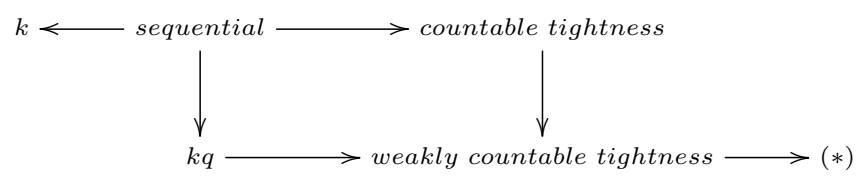

It is well-known that the inverses of implications above except for "countable tightness $\rightarrow$ weakly countable tightness", " $k q \rightarrow$ weakly countable tightness", and "weakly countable tightness $\rightarrow(*)$ " need not be true in general (see [9],[12],[14] and [15]).

From the following examples, we see that every space having weakly countable tightness is neither a $k q$-space nor a space having countable tightness and every space satisfying property $(*)$ need not have weakly countable tightness in general.

Example 2.15. In Example 2.7, we showed that the space $X$ does not have countable tightness. Since every countable subset of $X$ is closed, it is easy to check that for each subset $A$ of $X, A=[A]_{c}$; i.e., $A$ is a strongly sequentially closed set in $X$. And we show that a subset $K$ of $X$ is compact if and only if $K$ is finite. Suppose that there exists a compact subset $K$ of $X$ such that $|K| \geqq \omega$. Either $p \notin K$ or $p \in K$. If $p \notin K$, then since $K=\bigcup\{\{x\}: x \in K\}$ and $\{x\}$ is open for each $x \in K$, $K$ is not compact. This is is a contradiction. If $p \in K$, then there exists a subset $A$ of $K$ such that $p \notin A,|A|=\omega$ and $|K \backslash A| \geqq \omega$. Hence, $p \in X \backslash A$ and $X \backslash A$ is open. Clearly,

$$
K=A \cup(K \backslash A) \subset(\bigcup\{\{x\}: x \in A\}) \cup(X \backslash A) .
$$

So, we see that $\{\{x\}: x \in A\} \cup\{X \backslash A\}$ is an open over of $K$. Since $K$ is compact, there exists a finite subcover. But, there does not exist any finite subcover. This is a contradiction. The converse is trivial. Now we claim that $X$ has weakly countable tightness. Let $A$ be a strongly sequentially closed set in $X$ and $K$ a compact subset of $X$. Then by the fact shown above, $A \cap K$ is finite and hence closed. Therefore, $X$ has weakly countable tightness, but does not have countable tightness.

Example 2.16. Let $\beta \mathbb{N}$ be the Stone-Cech compactification of the positive integer set $\mathbb{N}$ with the discrete topology, $p$ is an element of $\beta \mathbb{N} \backslash \mathbb{N}$ and $q \notin \beta \mathbb{N}$. Let $X=\mathbb{N} \cup\{p, q\}$ with a topology as follows: a subset $U$ of $X$ is open in $X$ if either $q \in U$ and $X \backslash U$ is finite or $U$ is open in the subspace $\mathbb{N} \cup\{p\}$ of $\beta \mathbb{N}$. Then clearly the space $X$ is a $T_{1}$-space having countable tightness since $X$ is countable, and hence $X$ has weakly countable tightness. In [9, Example 2.4], the author 
showed that $X$ is compact and $\mathbb{N} \cup\{q\}$ is sequentially closed in $X$, but $\mathbb{N} \cup\{q\}(=(\mathbb{N} \cup\{q\}) \cap X)$ is not closed. Thus, we see that $X$ is not a $k q$ -space.

Example 2.17. The space of ordinals $X=\left[0, \omega_{1}\right]$, in Example 2.6, satisfies property $(*)$. Note that $\left[0, \omega_{1}\right)$ is a strongly sequentially closed set in $X$. Since $X$ is compact and and $\left[0, \omega_{1}\right)\left(=\left[0, \omega_{1}\right) \cap X\right)$ is not closed in $X,\left[0, \omega_{1}\right)$ is not a $k$-closed set in $X$ and hence $X$ does not have weakly countable tightness.

From definitions, we have immediately the following theorem and hence we omit the proof.

Theorem 2.18. If a space $X$ is a $k$-space having weakly countable tightness, then $X$ has countable tightness.

Remark 2.19. It is well-known that the subspace $X=\mathbb{N} \cup\{p\}$ of $\beta \mathbb{N}$, in Example 2.16, is not a $k$-space (see [15, Sec. 8.3, Problems 103]). Obviously, $X$ has countable tightness. Hence, we know that the converse of Theorem 2.18 need not be true. Note that a $k$-space does not have countable tightness in general (see [11, Example 2.7]).

Theorem 2.20. If a countably compact and submaximal space $X$ has countable tightness, then $X$ is a $k$-space.

Proof. Suppose that it is not. Then there exists a subset $A$ of $X$ such that for each compact subset $K$ of $X, A \cap K$ is closed and $A$ is not closed. By Lemma 2.9, $|\bar{A} \backslash A|<\aleph_{0}$, let $\bar{A} \backslash A=\left\{x_{1}, x_{2}, \ldots, x_{n}\right\}$. Since $X$ has countable tightness, there are subsets $C_{1}, C_{2}, \ldots, C_{n}$ of $X$ such that for each $i \in\{1,2, \ldots, n\}, C_{i}$ is countable and $x_{i} \in \overline{C_{i}}$. Let $D=\bigcup\left\{C_{i}: 1 \leqq i \leqq n\right\}$. Then clearly

$\bar{D}=\bigcup\left\{\overline{C_{i}}: 1 \leqq i \leqq n\right\}=\left(\bigcup\left\{C_{i}: 1 \leqq i \leqq n\right\}\right) \cup\left(\bigcup\left\{\overline{C_{i}} \backslash C_{i}: 1 \leqq i \leqq n\right\}\right.$.

By Lemma 2.9, for each $i \in\{1,2, \ldots, n\},\left|\overline{C_{i}} \backslash C_{i}\right|<\aleph_{0}$ and hence $|\bar{D}| \leqq$ $\aleph_{0}$. Since $X$ is countably compact, $\bar{D}$ is countably compact. It is easy to check that every countable and countably compact space is compact. Thus, $\bar{D}$ is compact. And we see that for each $i \in\{1,2, \ldots, n\}, C_{i} \subset A \cap \bar{D}$ and so $x_{i} \in \overline{C_{i}} \subset \overline{A \cap \bar{D}}$. Hence we have that if $A \cap \bar{D}$ is closed, then $x_{i} \in A \cap \bar{D}$ for each $i \in\{1,2, \ldots, n\}$ and so $x_{i} \in A$ for each $i \in\{1,2, \ldots, n\}$. It is impossible. Thus $A \cap \bar{D}$ is not closed, which is a contradiction.

From Theorems 2.18 and 2.20, we obtain directly the following corollary and hence omit the proof. 
Corollary 2.21. Let $X$ be a countably compact and submaximal space. Then $X$ has countable tightness if and only if $X$ is a $k$-space having weakly countable tightness.

We now show properties of a space having weakly countable tightness.

Theorem 2.22. Every closed subspace of a space having weakly countable tightness has weakly countable tightness.

Proof. Suppose that there exists a closed subspace $Y$ of a space $X$ having weakly countable tightness such that $Y$ does not have weakly countable tightness. Then since $Y$ does not have weakly countable tightness, there are a strongly sequentially closed subset $A$ of $Y$ and a compact subset $K$ of $Y$ such that $A \cap K$ is not closed. Note that since $Y$ is closed in $X,[A]_{c}^{X}=[A]_{c}^{Y}$, where $[A]_{c}^{X}\left([A]_{c}^{Y}\right)$ is the c-closure of $A$ in $X($ resp. in $Y)$. And we have clearly that $K$ is compact in $X$ and $A \cap K$ is not closed in $X$. It follows that $X$ does not have weakly countable tightness. This is a contradiction.

Lemma 2.23. Let $f: X \rightarrow Y$ be a continuous and surjective function from a space $X$ onto a space $Y$. If $B$ is a strongly sequentially closed set in $Y$, then $f^{-1}(B)$ is a strongly sequentially closed set in $X$.

Proof. Suppose that it is not and let $x \in\left[f^{-1}(B)\right]_{c}^{X} \backslash f^{-1}(B)$. Then there exists a countable subset $C_{x}$ of $f^{-1}(B)$ such that $x \in \overline{C_{x}}$. Clearly, $f\left(C_{x}\right)$ is a countable subset of $B$ and $f(x) \in f\left(\overline{C_{x}}\right)$. Since $f$ is continuous, $f\left(\overline{C_{x}}\right) \subset \overline{f\left(C_{x}\right)}$ and so $f(x) \in \overline{f\left(C_{x}\right)}$. Hence, $f(x) \in[B]_{c}^{Y}$. By hypothesis; $B=[B]_{c}^{Y}$, we have $f(x) \in B$; i.e., $x \in f^{-1}(B)$. This is a contradiction.

Theorem 2.24. Let $f: X \rightarrow Y$ be a continuous, open and surjective function from a space $X$ having weakly countable tightness onto a space $Y$ satisfying for each open set $U$ in $X, f^{-1}(f(U))=U$. Then $Y$ has weakly countable tightness.

Proof. Suppose that there exist a strongly sequentially closed subset $B$ of $Y$ and a compact subset $K$ of $Y$ such that $B \cap K$ is not closed. It is easy to show that $f^{-1}(K)$ is compact in $X$ by hypothesis for $f$. By Lemma $2.23, f^{-1}(B)$ is a strongly sequentially closed subset of $X$. Since $X$ has weakly countable tightness, $f^{-1}(B) \cap f^{-1}(K)$ is closed. Since $f^{-1}(B) \cap f^{-1}(K)=f^{-1}(B \cap K)$ and $f$ is open and surjective, $f\left(f^{-1}(B) \cap\right.$ $\left.f^{-1}(K)\right)=B \cap K$ and $B \cap K$ is closed. This is a contradiction. 


\section{References}

[1] A. V. Arhangel'skii and L. S. Pontryagin (Eds.), General Topology I, Encyclopaedia of Mathematical Sciences, vol. 17, Springer-Verlage, Berlin, 1990.

[2] A. V. Arhangel'skii, Topological Function Spaces, Mathematics and Its Appl. (Soviet Series), vol. 78, Kluwer Academic Publ., London, 1992.

[3] A. V. Arhangel'skii and P. J. Collins, On submaximal spaces, Topology and its Appl. 64(1995), 219-241.

[4] J. Dugundji, Topology, Allyn and Bacon, Inc., Boston, 1970.

[5] S. P. Franklin, Spaces in which sequences suffice, Fund. Math. 57(1965), 107-115.

[6] H. Z. Hdeib, On spaces which has countable tightness, Questions Answers Gen. Topology 6(1)(1988), 11-20.

[7] W. C. Hong, Generalized Fréchet-Urysohn spaces, J. Korean Math. Soc. 44(2)(2007), 261-273.

[8] W. C. Hong, A note on spaces which have countable tightness, Commun. Korean Math. Soc., 26(2)(2011), 297-304.

[9] S. Lin and C. Zheng, The k-quotient images of metric spaces, Commun. Korean Math. Soc., 27(2)(2012), 377-384.

[10] M. A. Moon, M. H. Cho and J. Kim, On AP spaces concerning with compact-like sets and submaximality, Comment. Math. Univ. Carolin. 52(2)(2011), 293-302.

[11] T. W. Rishel, A class of spaces determined by sequences with their cluster points, Portugal. Math. 31(1972), 187-192.

[12] F. Siwiec, Generalizations of the first axiom of countability, Rocky Mountain J. Math. 5(1)(1975), 1-60.

[13] L. A. Steen and J. A. Seebach, Jr., Counterexamples in topology, Springer-Verlag, Berlin, 1978.

[14] Y. Tanaka, Necessary and sufficient conditions for products of k-spaces, Topology Proceedings, 14(1989), 281-313.

[15] A. Wilansky, Topology for Analysis, Ginn and Company, 1970.

\section{Woo Chorl Hong}

Department of Mathematics Education, Pusan National University, Pusan 609-735, Korea.

E-mail:wchong@pusan.ac.kr 